\title{
El uso de las redes sociales en las ONG inmigrantes en España
}

\author{
Elias Said Hung, PhD \\ Doctor en Ciencias de la Información (Universidad Complutense de Madrid) \\ Docente investigador de la Universidad del Norte \\ Barranquilla, Colombia \\ saide@uninorte.edu.co \\ Jorge Valencia Cobos, BA \\ Universidad del Norte \\ Alumno de Maestría en Educación (Universidad Del Norte) \\ Barranquilla, Colombia \\ javalenciac@uninorte.edu.co
}

\begin{abstract}
Resumen
Este artículo tiene como objetivo analizar el uso de las redes sociales por las ONG que trabajan con población inmigrante en España. Para ello, se analizaron 18 ONG, con canales Twitter y Facebook y portales web; asimismo se analizó un total de 647 mensajes publicados por estos, durante 2010 y 2011. Los datos muestran un bajo nivel de apropiación de las redes sociales por parte de las ONG analizadas, así como el carácter informativo y poco participativo en que son empleados estos espacios actualmente; lo que reafirmaría lo expuesto por Albaigès en cuanto a la falta de elementos para un uso oportuno del término "cooperación 2.0", desde las organizaciones analizadas.
\end{abstract}

Palabras clave: redes sociales, ONG, inmigración, participación, cooperación 2.0.

\section{Introducción}

$\mathrm{T}$ al como expone Herrera (1998), al hablar del Tercer Sector, muchos relacionan a este con el voluntariado que las personas suelen idealizar, puesto que asisten a determinados grupos sociales en condición de vulnerabilidad. Según este autor, existen diferentes tipos de organizaciones enmarcadas en este sector: 1) organizaciones de caridad no gubernamental y no lucrativa, con un perfil filantrópico y tradicional (ejemplo: Dividendos Por Colombia); ${ }^{1}$ 2) organizaciones sociales no gubernamentales y no lucrativas, orientadas a áreas prioritarias de intervención a nivel social (educación, salud, religiosas, científicas y culturales, caritativas, entre otras), como por ejemplo, Médicos Sin Fronteras; ${ }^{2}$ 3) organizaciones políticas no gubernamentales y no lucrativas, orientadas a la concreción de un fin público o cuasi público (por ejemplo, Fundación Pablo Iglesias); ${ }^{3}$ y 4) organizaciones no gubernamentales y no lucrativas de comercio, orientadas a la consecución de objetivos comerciales no empresariales (por ejemplo, Fundación Copade). ${ }^{4}$

\footnotetext{
1. Disponible en: <http://www. dividendoporcolombia.org/>.

2. Disponible en: <http://www.msf.es/>.

3. Disponible en: <http://www.fpabloiglesias.es/>.

4. Disponible en: <http://www.copade.es/>.
} 


\section{Las redes sociales como espacios de participación ciudadana}

De acuerdo con Albaigès (2007), las TIC deben ser consideradas como un rasgo siempre presente en las sociedades contemporáneas. Pese al crecimiento del Tercer Sector, ${ }^{5}$ en las últimas décadas (Gui, 1990; Ambrosini, 1999; Herrera, 1998), persiste un horizonte inmaduro de desarrollo y aplicación de las TIC en él (Acevedo, 2004).

Autores como Joyanes (1997), Castells (2000) o Cardoso (2008) señalan que internet, no solo se ha convertido en una autopista de la información (cada vez más compleja), sino también en un escenario que brinda a sus usuarios una comunicación más directa y transparente, en el momento de compartir experiencias, conocimientos e intereses, de primera mano y editada por ellos mismos. Ello, por medio del auge de las redes sociales (ejemplo: Twitter y Facebook), en las que las comunidades digitales cuentan con la capacidad de generar materiales comunicables (Sánchez, 2008), en los que trasciende la desconfianza hacia la cosmovisión planteada desde los poderes o actores tradicionales, a través de la utilización de estos nuevos espacios digitales, que ayudan a la generación de mecanismos de contestación social y acceso a información transparente y sin intermediarios (Cremades, 2007).

Bajo el contexto descrito hasta ahora, la e-ciudadanía y la ciberdemocracia reposan bajo el ideal del "individuo en red", caracterizado por estar más informado y con mayores oportunidades de acceso a una información no manipulada por los medios tradicionales. Este escenario hace que resulte importante considerar un conjunto de aspectos, tanto a nivel individual como institucional, orientados a la promoción de alguno de los fines que tienen las ONG (Tabla 1).

El contexto actual exige el fortalecimiento del rol de los actores públicos asumidos en las labores ya indicadas; así como la promoción de nuevos mecanismos de entrada a grandes mayorías de individuos desde las ONG, a través de los nuevos espacios digitales (Sierra y Del Valle, 2007). Lo aquí señalado se enmarca en los planteamientos de Hoff, Horrocks y Tops (2000), Subirats (2002) y Harto (2006), en lo que se refiere al desarrollo de mecanismos de promoción de la democracia electrónica, a través de la aplicabilidad de estrategias orientadas al fortalecimiento de políticas de uso de las TIC, a favor de redes pluralistas de prestación de servicios, y de los procesos que contribuyan a la democracia directa.
Tabla 1 - Principales fines de los actores del Tercer Sector

La lucha a favor de la generación de mecanismos de inclusión social desde internet.

La lucha para la reducción de los procesos de exclusión social que aún presentan muchos individuos y países, al hacer uso y tener acceso a internet.

La promoción de mecanismos de discusión pública de temas de interés colectivos, por parte de los diferentes actores que ejercen su acción dentro de nuestras sociedades.

El aumento del compromiso público "online" de las prácticas ejercidas por estas organizaciones y desde las mismas, de acuerdo con lo expuesto por Araya (2005).

El aumento de la calidad de los mecanismos de participación ciudadana desde internet y las redes sociales, por ejemplo (Martí, 2008).

El fomento de las capacidades individuales y colectivas de interconexión para un uso pragmático y creativo del ecosistema de interacción y cultural promovido desde el uso de las TIC y las redes sociales (Sierra y DelValle, 2007).

El fomento de redes de comunicación, organización y de acción social que garantice la creación de espacios democrático-autónomos.

El aumento de la conectividad social que permita la articulación de una masa crítica de ciudadanos bajo la mediación tecnológica (Sierra y DelValle, 2007).

La puesta a disposición de espacios que ayuden al ciudadano a acceder a información veraz.

El uso significativo de las TIC, en el que deben ser pensadas como un medio al servicio de la participación ciudadana y la democracia deliberativa aquí expuesta.

La promoción de mecanismos que garanticen el intercambio de la deliberación pública y democrática a nivel social.

Fuente: Elaborado por los autores.

El contexto actual exige el fortalecimiento del rol de los actores públicos asumidos en las labores ya indicadas; así como la promoción de nuevos mecanismos de entrada a grandes mayorías de individuos desde las ONG, a través de los nuevos espacios digitales (Sierra y Del Valle, 2007). Lo aquí señalado se enmarca en los planteamientos de Hoff, Horrocks y Tops (2000), Subirats (2002) y Harto (2006), en lo que se refiere al desarrollo de mecanismos de promoción de la democracia electrónica, a través de la aplicabilidad de estrategias orientadas al fortalecimiento de políticas de uso de las TIC, a favor de redes pluralistas de prestación de servicios, y de los procesos que contribuyan a la democracia directa.

5. Caracterizadas principalmente, de acuerdo con Ambrosini (1999), por: una amplia variedad de actividades y dimensiones en que se organizan internamente; una diversificación de las fuentes de financiamiento; así como por la capacidad sistemática de articulación de convivencia de espacios colaborativos con actores públicos y la diversidad de estatus jurídicos en ellas. 


\section{La cooperación 2.0}

En las sociedades contemporáneas parece existir un amplio consenso del impacto que pueden traer consigo las TIC (Peña-López, 2008). La Declaración del Milenio hace una exposición explícita de la necesidad de "velar por que todos puedan aprovechar los beneficios de las nuevas tecnologías" (Naciones Unidas, 2000, p. 6). Según McConnell (1995), las TIC cuentan con la capacidad de generar un contexto, en el que se promueva una mejora de la gestión de los recursos informativos a nivel social; y una mayor comprensión de los potenciales beneficios generados por estas, en la mejora del diseño de los sistemas y servicios de información y comunicación a nivel social.

Bajo el concepto de Cooperación 2.0, se han logrado aplicar acciones orientadas a nutrir y catalizar procesos desde las ONG, a favor de la plena integración de estos recursos para la promoción, planificación y ejecución de actividades, proyectos, programas y planes relacionados con los fines dispuestos por este tipo de organizaciones y para la población atendida o beneficiaria de estas (Labelle, 2003).

La concepción alrededor de la Cooperación 2.0 debe trascender la mera aplicación o instrumentalización de la tecnología, para comprenderla como un proceso orientado a la re-ingeniería de la cooperación. En otras palabras, a una manera diferente de llevar a cabo esta actividad, a través del empoderamiento de las TIC. Ello como resultado de las responsabilidades de los miembros de este tipo de organizaciones, en el momento, según Acevedo (2004, 2006), de: 1) buscar la mejor eficacia en sus acciones o actividades, así como los recursos dispuestos para tales fines; 2) generar mecanismos de control de cómo actúan; 3) garantizar el efecto catalizador de las TIC que ayude a la generación de acciones más extensas; y 4) garantizar la posibilidad de compartir, intercambiar y participar, bajo un contexto más horizontal, tanto con otros actores a nivel social, como con sus potenciales beneficiarios y miembros de equipo.

A pesar del marco de responsabilidades que tienen las ONG para la mayor aplicabilidad de las TIC en sus funciones, se sigue observando una falta de familiaridad en las posibilidades de estas; detectándose además un conjunto de debilidades que inciden en ello, las cuales, de acuerdo con Berrios (2009), son: 1) la falta de recursos en las instalaciones requeridas para el uso efectivo de las TIC; 2) la falta de promoción de medidas que ayuden a la reducción de las brechas digitales observadas en el interior de muchas ONG; 3) la falta de mecanismos de formación del capital humano en el interior de muchas organizaciones en el desarrollo de competencias orientadas al uso efectivo de los avances tecnológicos; y 4) la falta de planifica- ción de procesos de inclusión de las TIC en el ejercicio de sus funciones o misiones a nivel social.

En la actualidad, de acuerdo con autores como Acevedo (2004) y Berrios (2009), los actores vinculados con el Tercer Sector en España se caracterizan por un avance lento, insuficiente, esporádico y, en especial, carente de una estrategia clara para el aprovechamiento de las TIC; superando muchas veces lo discursivo sobre lo práctico al respecto. Ello, motivado por una falta de comprensión del papel que puede jugar este tipo de recursos, en el momento de aplicar los espacios y recursos digitales para la promoción de mecanismos de participación, contacto, visibilidad y gestión de las acciones planteadas por entidades como las ONG.

Por tanto, el término Cooperación 2.0 parece muy alejado de la realidad actual. Sobre todo si asumimos como válidos los señalamientos dados por este Albaigès (2007) en lo que se refiere a la presencia de muchas ONG "0.0", es decir, carentes de portales Web o de una estática y desactualizada; y en el mejor de los casos de "entidades 1.0"; incapaces de sacar máximo provecho de las TIC y de los espacios digitales actualmente existentes, desde el punto de vista de Acevedo (2006), Rischard (2003), tal como se muestra en la Tabla 2.

Tabla 2 - Objetivos orientados al aprovechamiento de las TIC, según Acevedo (2006) y Rischard (2003)

\begin{tabular}{|l|l|}
\hline $\begin{array}{l}\text { La simplificación y reducción } \\
\text { de las tareas mecánicas } \\
\text { realizadas por estas. }\end{array}$ & $\begin{array}{l}\text { El acceso fácil y rápido } \\
\text { de la información del } \\
\text { personal a cargo dentro de } \\
\text { la organización. }\end{array}$ \\
\hline $\begin{array}{l}\text { La adecuación de los } \\
\text { procesos de gestión de } \\
\text { los diferentes recursos de } \\
\text { conocimiento e información. }\end{array}$ & $\begin{array}{l}\text { El cumplimiento de los } \\
\text { objetivos propuestos en } \\
\text { los diferentes proyectos } \\
\text { ejecutados por estas } \\
\text { entidades. }\end{array}$ \\
\hline $\begin{array}{l}\text { El fortalecimiento de las } \\
\text { capacidades humanas e } \\
\text { institucionales. }\end{array}$ & $\begin{array}{l}\text { El aumento de } \\
\text { mecanismos de } \\
\text { participación. }\end{array}$ \\
\hline $\begin{array}{l}\text { El uso eficaz de las TIC para } \\
\text { la producción y divulgación } \\
\text { del conocimiento generado } \\
\text { desde los proyectos realizados } \\
\text { por estos. }\end{array}$ & $\begin{array}{l}\text { El fomento de un } \\
\text { contexto de actividad, } \\
\text { desde las ONG, orientadas } \\
\text { a generar soluciones que } \\
\text { ayuden al desarrollo de } \\
\text { temáticas, a mejorar sus } \\
\text { acciones y contextos con } \\
\text { potenciales donantes y } \\
\text { promover un cambio de } \\
\text { mentalidad orientado a } \\
\text { interiorizar e el paradigma } \\
\text { de la economía basado en } \\
\text { la Sociedad en Red. }\end{array}$ \\
\hline
\end{tabular}

Fuente: Elaborado por los autores. 
Metodología

El artículo parte de un enfoque de investigación cuantitativo, orientado a: la caracterización, cuantificación y evaluación del uso de las redes sociales virtuales para la promoción de la participación ciudadana en el contexto de las ONG, que trabajan con población inmigrante en España. ${ }^{6}$

Teniendo en cuenta las características propias del fenómeno estudiado aquí (la participación ciudadana desde las redes sociales), se consideraron dos unidades operativas de análisis: el sitio web de la ONG y los mensajes publicados.

Las unidades muestrales tomadas para el estudio fueron seleccionadas con base en los siguientes criterios: 1) que actuaran de forma directa o indirecta en territorio español, con alguno de sus ejes de actuación en torno a la población inmigrante; 2) ONG que estuviesen registradas en la GuíaOngs, ${ }^{7}$ existente en España; 3) ONG que poseyeran, en el momento del levantamiento expuesto en este artículo, un portal o equivalente (blog por ejemplo) técnicamente activo; y 4) ONG que dispusieran de canales o usuarios activos tanto en Twitter como en Facebook.
En una primera etapa de selección de la muestra, se lograró identificar un total de 295 ONG registradas en mayo de 2010 en GuiaONGs. ${ }^{8}$ En esta exploración inicial, se logró identificar un total de 176 ONG que cumplían con los criterios expuestos en el párrafo anterior, así como con los requerimientos de levantamiento de los datos definidos para esta población de estudio: 1) información institucional de las ONG y fundaciones estudiadas aquí; y 2) uso de recursos web por parte de las ONG y fundaciones abocadas al trabajo social orientado a la inmigración en España.

De las 176 ONG tomadas para el análisis general de la información institucional, solo 18 tenían o hacían uso de Twitter y Facebook (tabla 3). Dichas ONG (10\% del total de las ONG identificadas aquí) fueron tomadas como muestra de caso tipo en el momento de medir aspectos como: 1) el perfil de la visibilidad de las redes sociales en los portales web de las ONG; 2) el perfil de las ONG en Twitter; 3) el perfil de las ONG en Facebook; y 4) el perfil de usuarios y los mensajes transmitidos desde los canales en Twitter y Facebook de las ONG.

Cada una de las unidades muestrales fue analizada a través de algunas de las variables e indicadores expuestos en la tabla 4.

Tabla 3 - ONG que trabajan con población inmigrante en España, con posesión de canales Twitter y Facebook, según la GuíaONg en marzo de 2010

\begin{tabular}{|l|l|l|l|}
\hline Nombre & URL & Nombre & URL \\
\hline ACOLVALLE & http://acolvalle.org/ & Fundación Altius-UFV & http://www.1kilodeayuda.es/ \\
\hline Alza tu voz & http://www.alzatuvoz.es/ & Fundación Entreculturas & http://www.entreculturas.org/ \\
\hline AOSLA-Gizalan & http://www.aosla.org/ & Fundación Luis Vives & http://www.fundacionluisvives.org/ \\
\hline $\begin{array}{l}\text { Asamblea de } \\
\text { Cooperación por la Paz }\end{array}$ & http://www.acpp.com/ & Fundación Pere Tarré & http://www.peretarres.org/ \\
\hline Ashoka Spain & http://www.ashoka.es/ & GATS & http://www.gats.cat/ \\
\hline Cáritas Española & http://www.caritas.es/ & Jóvenes Verdes & http://www.jovenesverdes.org/ \\
\hline EAPN-ES & http://www.eapn.es/ & Movimiento por la Paz & http://www.mpdl.org/ \\
\hline Federació Valenciana & http://www.faavem.org/ & Red Araña & http://www.empleoenred.org/ \\
\hline Fundación A.D.R.A. & http://www.adra-es.com/ & Teléfono de la Esperanza & http://www.telefonodelaesperanza.org/ \\
\hline
\end{tabular}

Fuente: Elaborado por los autores.

Tabla 4 - Principales variables y categorías consideradas

Año de constitución de la ONG; presencia de icono de redes sociales en portal web; audiencia mensual de la Web institucional; uso de redes sociales (Twitter y Facebook); presencia en otras redes sociales; tiempo de apertura de canales en Twitter y Facebook; número de tweets en el muro publicados por la ONG; número de seguidores de la ONG; mensaje publicado en canal de la ONG; tipo de mensaje publicado en la red social de ONG.

Fuente: Elaborado por los autores.

6. El perfil medio de la inmigración en España se caracterizaba en 2010, según Moreno y Bruquetas (2010), por: 1) representar el 12,17 \% de la población total de este país; 2) tener una edad promedio de 32,6 años; 3) ser mayoritariamente masculina (52,9 \% del total existente); 4) ser originarios, en su mayoría, de Europa del Este, África y América Latina; 5) poseer una tasa mayor de paro (desempleo) que los observados entre los españoles; 6) dedicarse en su mayoría al trabajo agrario y doméstico; y 7) poseer menores niveles de estudios, en comparación con los nacionales de este país, a nivel superior (salvo en el caso de los asiáticos y los inmigrantes del UE25-sin España; entre algunos de los rasgos que destacar de estos

7. La selección de esta guía se hizo por ser uno de los listados existentes en España y de acceso público con un mayor número de ONG registradas en España, y porque además permitía la identificación, según la población atendida, de las ONG dispuestas en esta guía.

8. Disponible en: <http://www.GuiaONGss.org/>. 
Los indicadores tomados como referencia para el desarrollo de este trabajo (tabla 4) fueron analizados a partir de la extracción directa de los datos desde Twitter y Facebook; a través de indicadores de tipo cuantitativos, dispuestos en: Twerpscan, Tweetstat, Twitalyzer, TwitterCounter y RetweetRank, entre otras. ${ }^{9}$

Sobre los mensajes (tweets y mensajes en el muro), publicados desde los canales abiertos por ONG en Twitter y Facebook, se aplicó la técnica de análisis de contenido. La selección de los mensajes se realizó en tres periodos distintos: marzo de 2010, septiembre de 2010 y marzo de 2011. Ello, con el fin de abarcar momentos distintos de tiempo, que ayudasen a aislar los posibles hechos coyunturales que contribuyesen a posibles sesgos del contenido expuestos en los mensajes analizados. El número total de mensajes analizados en este estudio fueron: 239 mensajes en el muro de Facebook y 408 Tweets publicados en el conjunto de canales de Twitter y Facebook.

\section{Resultado}

\section{Perfil web de las ONG}

A partir de la tabla 5, podemos observar cómo las 18 ONG con canales en Twitter y Facebook, del total de 176 ONG de nuestro universo de estudio, poseían una trayectoria comprobada en materia de inmigración de más de veinticinco años. Asimismo, podemos ver como Twitter era la red social con mayor visibilidad desde sus portales web, ubicándose, en la mayoría de los casos analizados, en la zona superior de estos portales. En el caso de otras redes sociales (como por ejemplo Facebook o Youtube), la $\mu$ observada nos muestra que, en la mayoría de las ONG, estas poseen una visibilidad casi inexistente, desde los canales institucionales dispuestos (portales web). Esto hace que muchos de los potenciales interesados (población beneficiaria, por ejemplo) desconocieran la existencia de otros mecanismos de acceso a información relacionada con el trabajo realizado por estas organizaciones.

\section{Perfil de las ONG en Twitter}

Las tablas 5, 6 y 7, nos permiten distinguir los principales rasgos que tienen las ONG estudiadas en Twitter. En general, el rol asumido por estas organizaciones se enmarca en lo que Barone (2010) ha dado en denominar como everyday users, es decir, usuarios con un gran potencial de impacto en otros usuarios desde círculos pequeños de influencia construidos desde sus canales en Twitter; pero que además asumen roles propios de usuarios conectados con otros miembros de esta red social, con grandes competencias en el momento de comunicarse con estos.

En cuanto al perfil expuesto por las ONG estudiadas en Twitter (tabla 3), se observa que la zona biográfica es usada para exponer a otros usuarios el perfil institucional de cada organización.

Respecto al perfil de uso diario de las ONG en la red social tratada en este apartado, las tablas 5 y 7 nos muestran que la mayoría de estas organizaciones hacen uso de Twitter en horario y días laborales principalmente. Esto nos mostraría una falta de cumplimiento de uno de los rasgos propios de la Sociedad en Red: la posibilidad de "estar conectados" durante todo el día y establecer contacto en tiempo real con los diferentes seguidores de los usuarios. Por tanto, el manejo realizado por estas ONG de Twitter parece ser más afín a dinámicas propias de los medios de co-

Tabla 5 - Presencia de redes sociales en el portal Web de ONG con canales en Twitter y Facebook analizadas

\begin{tabular}{|c|c|c|c|c|c|}
\hline & Mínimo & Máximo & $\mu$ & Desv. típ. & $\mathrm{N}$ \\
\hline Tiempo de existencia de ONG (años) & 9 & 64 & 26,00 & 15,232 & 12 \\
\hline 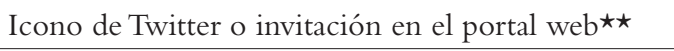 & 1 & 5 & 3,72 & 1,447 & 18 \\
\hline 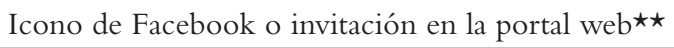 & 1 & 5 & 1,39 & 1,145 & 18 \\
\hline Presencia en otras redes sociales $\star$ & 1 & 2 & 1,44 &, 511 & 18 \\
\hline Presencia de canalYoutube ${ }^{\star}$ & 1 & 2 & 1,39 &, 502 & 18 \\
\hline
\end{tabular}

\section{Nota:}

$\star^{\mathrm{S} i ́}=1 / \mathrm{No}=2$

$\star \star$ No aparece=1 / Mitad Superior=2 / Mitad inferior=3 / Superior=4 / Inferior=5

Los casos perdidos son resultado de la falta de datos en el momento de levantar la información requerida para el desarrollo del estudio.

9. Como ejemplo de lo aquí expuesto tenemos el caso de RetweetRank y Twerpscan, los cuales hacen un análisis estadísticamente significativo, con un nivel de confianza del $97 \%$ y $\alpha=0,01$, de aproximadamente 12.000 Tweets del total publicado por los usuarios (RetweetRank) y de más del $95 \%$ de los Tweets enviados (Twerpscan) 
municación tradicionales que de los usuarios dentro de la esfera digital.

En lo que se refiere al uso diario que suelen hacer de Twitter, los canales abiertos por las ONG analizadas poseen un importante nivel de impacto entre los usuarios que le siguen en este espacio (tabla 5). Ello pese a la variabilidad observada, a través de la desviación típica de los datos expuestos en este artículo. Lo que permitiría intuir el tipo de mensajes expuestos por estos, los cuales parecen generar una fuerte conexión con un alto porcentaje de seguidores.

En lo que se refiere al número de seguidores (tabla 5), se observa una alta variabilidad entre las ONG analizadas. Pese a ello, podemos ver cómo la media de seguidores nos muestra un contexto en el que pudiésemos afirmar que el grupo de casos analizados en su conjunto tienen un importante número de seguidores interesados en los mensajes transmitidos por las ONG analizadas, pero, además, están dispuestos a establecer comunicación con éstas.

En cuanto al número de tweets publicados por las ONG estudiadas (tabla 5), se aprecia una alta varia- bilidad de mensajes publicados, de 2 mensajes hasta 3.798 mensajes en el momento de la medición de los datos aquí expuestos. En general, se aprecia cómo estas organizaciones suelen publicar un alto número de tweets al día, de los cuales pocos suelen estar orientados al establecimiento de contacto directo con otros usuarios, y un alto número, en cambio, suelen ser retweets o reproducción de otros mensajes no provenientes o elaborados por estas organizaciones.

En cuanto a los recursos empleados por las ONG para la publicación de tweets, la tabla 7 nos permite ver que un gran número de mensajes suelen publicarse desde la web, es decir, desde los canales habilitados desde esta red social, sin hacer un uso de $A P I .{ }^{10}$ Ello nos llevaría a enmarcar el contexto de accesibilidad de estas entidades bajo procedimientos, considerados tradicionales, mediados por el ordenador, en lugar de hacer un mayor aprovechamiento del contexto actual de accesibilidad a este tipo de espacios digitales (redes sociales), a través de soportes móviles o bien interconectando los contenidos expuestos desde sus portales web tradicionales, por ejemplo.

Tabla 5 - Datos descriptivos de los canales Twitter de las ONG analizadas

\begin{tabular}{|l|c|c|c|c|c|}
\hline Variable & Media & Mínimo & Máximo & Desviación típica & N \\
\hline Tipo de rol del usuario Twitter de la ONG & 1,1 & 1 & 2 & 0,2 & 18 \\
\hline $\begin{array}{l}\text { Tipo de información expuesta en zona Bio del } \\
\text { usuario }\end{array}$ & 2,2 & 1 & 3 & 0,5 & 18 \\
\hline Densidad expositiva semanal de usuario en Twitter $\star \star$ & 4,3 & 2 & 6 & 1,4 & 18 \\
\hline Nivel de impacto del usuario Twitter de la ONG & $81,7 \%$ & $40,0 \%$ & $100,0 \%$ & 15,9243 & 18 \\
\hline Número de seguidores en Twitter (followers) & 18995,83 & 14 & 334415 & 78720,197 & 18 \\
\hline Número de seguidos en Twitter (following) & 6481,39 & 13 & 112200 & 26384,704 & 18 \\
\hline Número de tweets & 720,06 & 2 & 3798 & 1149,122 & 18 \\
\hline Porcentaje de retweet por usuarios & $16,18 \%$ &, $92 \%$ & $44,00 \%$ & 10,88 & 16 \\
\hline Porcentaje de replies por usuarios & $6,127 \%$ &, $90 \%$ & $15,00 \%$ & 3,90 & 15 \\
\hline Media de tweets publicados por usuario al día & 29,12 & 1 & 76 & 23,108 & 17 \\
\hline
\end{tabular}

Nota:

$\star 1=$ Everyday Users; $2=$ Reporters

$\star \star 1=$ Mensaje personal; $2=$ Mensaje de tipo profesional/institucional; $3=$ Ningún mensaje

$\star \star \star 1=12$ am a 3:59 am; 2=4:00 am a 7:59 am; 3=8:00 am a 11:59 am; 4= $12 \mathrm{~m}$ a 3:59 pm; 5= 4:00 pm a 7:59 pm; 6= 8:00 pm a 11:59 pm Los casos perdidos son resultado de la falta de datos en el momento de levantar la información requerida para el desarrollo del estudio

Tabla 6 - Descriptivos de publicación de tweets por ONG estudiadas, según día de la semana

\begin{tabular}{|l|r|r|c|c|c|}
\hline \multicolumn{1}{|c|}{ Estadísticos descriptivos } & Media & Desv. típ. & Mínimo & Máximo & N \\
\hline Tweets agregados el domingo & 9,72 & 16,341 & 0 & 66 & 18 \\
\hline Tweets agregados el lunes & 116,33 & 183,384 & 0 & 613 & 18 \\
\hline Tweets agregados el martes & 123,44 & 195,907 & 0 & 623 & 18 \\
\hline Tweets agregados el miércoles & 155,28 & 261,814 & 0 & 933 & 18 \\
\hline Tweets agregados el jueves & 144,72 & 245,284 & 1 & 817 & 18 \\
\hline Tweets agregados el viernes & 94,56 & 145,812 & 1 & 522 & 18 \\
\hline Tweets agregados el sábado & 12,89 & 18,227 & 0 & 60 & 18 \\
\hline
\end{tabular}

Fuente: Elaborado por los autores.

10. Conocidos en castellano como Interfaz de programación de aplicaciones (IPA) o API (del inglés Application Programming Interface), son aplicaciones que permitan a los usuarios en internet compartir contenidos existentes en diferentes portales, así como el acceso a estos desde diferentes soportes. 
Tabla 7 - Descriptivos de publicación de tweets por ONG analizadas desde Twitter, según recursos o aplicaciones empleadas

\begin{tabular}{|l|c|c|c|c|c|}
\hline Estadísticos descriptivos & Media & Desv. típ. & Mínimo & Máximo & N \\
\hline Tweets enviados por Web por usuario & $\mathbf{2 5 8 , 3}$ & 443 & 2 & 1476 & 18 \\
\hline Tweets enviados por API Web y Movil por usuario & 34,1 & 134 & 1 & 569 & 18 \\
\hline Tweets enviados por API web por usuario & 17,1 & 60 & 1 & 245 & 18 \\
\hline Tweets enviados por API Móvil & 3,9 & 15,5 & 1 & 64 & 18 \\
\hline
\end{tabular}

Fuente: Elaborado por los autores.

\section{Perfil de las ONG en Facebook}

El uso que hacen de Facebook resulta menor en términos de usuarios seguidores o fans, en vista de la media observada de estos, en el conjunto de canales analizados, durante el período de levantamiento de los datos expuestos en este artículo (tabla 8). El bajo número de seguidores detectados guarda semejanza en cuanto al nivel de actividad observada por las organizaciones dentro de sus canales en esta red social. Ello como resultado de la media observada en torno al número de mensajes publicados por la ONG, du- rante el período tomado de análisis; así como por el nivel de participación de los seguidores u otros usuarios en estos canales.

Asimismo, podemos decir que el perfil de estas organizaciones dentro de esta red social resulta pasiva, es decir, hacen un uso de este escenario digital para la transmisión y exposición unidireccional de información generada o de interés por cada entidad, sin transcender, en la mayoría de los casos, al empleo de mensajes o estrategias que ayuden a dinamizar y favorecer el diálogo y la participación con los diferentes seguidores o fans adscritos a sus canales (tabla 9).

Tabla 8 - Descriptivos de la actividad de los canales Facebook de las ONG analizadas

\begin{tabular}{|l|c|c|c|c|c|}
\hline Estadísticos descriptivos & Media & Desv. típ. & Mínimo & Máximo & N \\
\hline Amigos/fans en Facebook & 2403,50 & 4703,248 & 0 & 18293 & 18 \\
\hline Mensajes publicados por la ONG, durante el período de análisis & 16,28 & 15,5 & 1 & 60 & 18 \\
\hline Mensajes de Anuncio/Titular de la ONG, durante el período de análisis & 1,65 & 3,6 & 0 & 14 & 18 \\
\hline Mensajes de usuarios, durante el período de análisis & 0,54 & 1,1 & 0 & 4 & 18 \\
\hline $\begin{array}{l}\text { Comentarios en mensajes publicados en semana de análisis, durante el } \\
\text { período de análisis }\end{array}$ & 2,5 & 2,662 & 1 & 8 & 18 \\
\hline $\begin{array}{l}\text { Comentarios en Anuncio/Titular de la ONG, durante el período de } \\
\text { análisis }\end{array}$ & 0,4 & 1,14 & 0 & 5 & 18 \\
\hline Comentarios en Mensajes de Usuarios, durante el período de análisis & 0,11 & 0,44 & 0 & 2 & 18 \\
\hline
\end{tabular}

Fuente: Elaborado por los autores.

Tabla 9 - Porcentajes de tipo mensajes, participación y gusto de los mensajes publicados desde los canales de Facebook de las ONG estudiadas

\begin{tabular}{|c|c|c|}
\hline Tipo de Mensaje (Facebook) & Total & $\%$ \\
\hline Anuncio/Titular de la ONG & 237 & 85,56 \\
\hline Mensajes de Usuarios & 39 & 14,1 \\
\hline Otros & 1 & 0,36 \\
\hline Total & 277 & 100 \\
\hline Tipo de participación (Facebook) & Total & $\%$ \\
\hline La ONG participa en los comentarios con intervenciones & 11 & $4 \%$ \\
\hline Los usuarios publican mensajes en el muro del medio & 45 & $16 \%$ \\
\hline No hay participación & 221 & $80 \%$ \\
\hline Total & 277 & $100 \%$ \\
\hline Gusto (Facebook) & Total & $\%$ \\
\hline Anuncio/Titular de la ONG & 3640 & 88,99 \\
\hline Mensajes de Usuarios & 447 & 10,92 \\
\hline Otros & 3 & 0,073 \\
\hline Total & 4090 & 100 \\
\hline
\end{tabular}

Fuente: Elaborado por los autores. 


\section{Perfil de usuarios y los mensajes transmitidos desde los canales en Twitter y Facebook de las ONG analizadas}

Los datos obtenidos en el marco de este trabajo nos permiten señalar que el $96 \%$ de los usuarios de Twitter (seguidores de estas $\mathrm{ONG}$ ) que publicaron comentarios durante el período de levantamiento de los datos aquí expuestos (175 de los 408 mensajes en total) poseían algún tipo de relevancia pública (local, nacional, regional, internacional o global). Esto nos lleva a identificar un escenario en el que parece que estos espacios digitales están siendo empleados más para el seguimiento o visibilidad de información o noticias, y otros referentes a sus fines misionales, que como espacios para el contacto y la participación con la población atendida por estas. Lo aquí expuesto se refuerza al ver cómo el $68 \%$ de los mensajes emitidos por estos usuarios fueron más de tipo informativos o noticiosos, mientras que el resto eran más de tipo de comentarios $(7 \%)$, procedimientos $(2 \%) \mathrm{u}$ otros de tipo personal (23\%).

Un alto porcentaje de los usuarios que participaron activamente desde los canales Twitter abiertos por las ONG analizadas residían en España y poseían nacionalidad española $(85,6 \%$, respectivamente), lo que nos llevaría a observar el marcado acento local o nacional que tienen estos espacios digitales, a pesar de llevar a cabo proyectos de cooperación orientados a la población inmigrante, desde sus países de origen (ejemplo: Teléfono de la Esperanza y Entreculturas, entre otros).

En cuanto al tipo de mensajes publicados desde los canales de Twitter de las ONG analizadas, vemos que casi la totalidad de estos mensajes analizados (408 mensajes en total) son de tipo informativo o noticioso $(92,9 \%)$, de tipo neutro $(82,1 \%)$ y emitido o replicado por la ONG (79,1\%).

En el caso de Facebook, el 57,4 \% de los usuarios (seguidores de estas $\mathrm{ONG}$ ) eran personas naturales, mientras que el resto (42,6\%) eran instituciones o miembros del Tercer Sector. Se observa además un contexto similar a lo visto en Twitter, ya que la mayoría de los usuarios analizados poseían nacionalidad española $44,4 \%$, mientras que no se pudo identificar la nacionalidad del 42,6\% de los mismos, al no disponer de datos o mecanismos de acceso de esta información desde esta red social; y el restante $13 \%$ de usuarios analizados, tenían nacionalidad brasileña, mauritana, norteamericana y mexicana.

En cuanto al lugar de residencia de los usuarios analizados en Facebook, en su mayoría indicaban como lugar de residencia a España (44,4 \%); para un
$40,7 \%$ no pudo establecerse datos sobre este tema, y el restante $14,9 \%$ residía en los países de origen de las nacionalidades antes expuestas. Por tanto, se mantendría lo expuesto en el caso de Twitter, es decir, el marcado acento local o nacional que parecen mantener las organizaciones dentro de estos espacios digitales.

Del total de mensajes publicados por los usuarios ajenos a las ONG, desde Facebook, un 31,5\% de los mensajes fueron agradecimientos y un $35,2 \%$, opiniones positivas sobre alguna información o noticia publicada en el muro de estas ONG. Mientras que el $33,3 \%$ de los restantes mensajes (54 de 239 mensajes en total) eran comentarios generales (13\%), sugerencias y opiniones negativas de una información o noticia publicada en el muro (9,3\%, respectivamente) y apenas un 1,9\% eran solicitudes de información por parte de estos usuarios. Por tanto, más que hablar de un escenario digital orientado a la participación de sus miembros y a la población interesada en la labor realizada por éstas, deberíamos hablar de contextos más informativos o expositivos, en los que estas entidades comparten noticias de interés general con otros usuarios.

Un cambio claro de lo visto en Facebook, en comparación con Twitter, fue la imposibilidad de poder determinar el carácter público o no de los usuarios participantes desde los canales abiertos por las ONG (57,4 \%); mientras que el 38,9\% de estos usuarios no tenía ningún tipo de relevancia pública y solo en un 3,7 \% si se logró establecer este tipo de rasgo. Si tomamos como referencia los porcentajes aquí mencionados, pareciera que los fines o usos de las organizaciones analizadas de Facebook pudiesen tener alguna variación respecto a Twitter, al menos en lo que se refiere a los fines empleados para cada caso y al claro aprovechamiento de Facebook con propósitos más personales y de contacto con entidades e individuos anónimos.

Además, vemos como el 96,7 \% de los mensajes publicados en el muro de los canales de las ONG analizadas (239 mensajes en total) provienen o son replicados por la misma organización; mientras que apenas un 3,3\% de mensajes son emitidos por un fan $\mathrm{u}$ otro usuario. Asimismo, los mensajes analizados nos permiten delinear un perfil de lo que suele exponerse en estos canales, predominado por mensajes de tipo informativo o noticioso $(92,9 \%$ del total de los 239 mensajes analizados) y neutros (87\% del total de los 239 mensajes analizados). Lo que nos llevaría a observar semejanzas con el caso de los canales de Twitter de estas ONG, empleándose rasgos similares entre sí, al utilizar esta red social como divulgadora o emisora de noticias e informaciones de interés de estas organizaciones, en la mayoría de los casos. 


\section{Conclusiones}

Los datos mostrados en este artículo nos permiten establecer un difícil contexto de uso efectivo del término Cooperación 2.0, en el interior de las ONG aquí analizadas. Si bien existen diversas declaraciones y acuerdos internacionales, en los que se destacan la importancia de las TIC en el desarrollo humano y para la reducción de las brechas sociales dispuestas en muchas sociedades contemporáneas (Naciones Unidas, 2000; PNUD, 2001; CEPAL, 2005); aún parecen distantes los impactos positivos que pueden brindar las TIC en el interior de las ONG analizadas para la concreción de dichos fines (Peña-López, 2008; McConnell, 1995).

La falta de espacios virtuales tradicionales (portales web), en un alto porcentaje de las ONG dispuestas en la GuiaONGs, dedicadas al trabajo con la población inmigrante en España (59 \% del total de las 296 ONG identificadas en este directorio contaban con portales web), y el reducido porcentaje de estas con presencia en las redes sociales en la actualidad (6 $\%$ del total de las 296 ONG identificadas en el directorio empleado), nos delinean un contexto de apropiación de las TIC, a nivel de internet, en el que se reafirmaría lo expuesto por Albaigès (2007), al menos en las ONG analizadas.

De acuerdo con lo descrito hasta ahora, el contexto de las ONG estudiadas contaría con claras dificultades en el momento de poder sacar provecho de las TIC, a favor de la promoción de contextos de comunicación más directa y transparente entre los diferentes actores que hacen parte de nuestras sociedades (Castells, 2000; Rischard, 2003; Acevedo, 2006; Cardoso, 2008; Cremades, 2007).

El escenario de predominio de ONG “0.0” ó "1.0" haría además que viésemos el escenario actual alejado o en contraposición al predominio que están teniendo las TIC en nuestras sociedades contemporáneas. Ello, en vista de que, al menos desde las en- tidades del Tercer Sector estudiadas, parecieran estar dominadas por un contexto de inmadurez y falta de conciencia de la importancia que revestiría, para la consecución de sus fines, un uso más acentuado de estos recursos (Acevedo, 2004).

El horizonte plasmado en el universo de estudio, a partir de la muestra analizada, hace que hablar de mecanismos de promoción de la participación ciudadana, desde los espacios digitales, por parte de estas entidades objeto de estudio, resultaría aún una idea no aplicada, al menos desde el punto de vista de autores como Acevedo (2004), Coleman y Gotze (2001), Bohman y Rehg (1997), Elster (1998), Macedo (1999) y Besson y Martí (2006), quienes establecen algunas incidencias que pueden traer consigo las TIC para el desarrollo de mecanismos que garanticen el diálogo y el contacto más directo entre las ONG analizadas y las poblaciones beneficiarias de estas (ciudadano), por ejemplo; así como en el momento de aplicar estrategias que contribuyan a la promoción de una democracia, en el sentido expuesto por Hagen (1997), Hoff, Horrocks y Tops (2000), Subirats (2002) o Harto (2006).

Por tanto, más que existir un contexto efectivo que contribuya a la promoción de la participación desde las redes sociales de las ONG analizadas, deberíamos decir que, en la actualidad, se requiere de acciones orientadas al fomento o la apropiación tecnológica, desde una perspectiva orientada al establecimiento de acciones que ayuden al cambio de mentalidad y estrategias hasta la fecha adoptadas. Ello, desde un paradigma más coherente y acercado a lo que hoy se conoce como Sociedad en Red, basado en la economía fundada en el conocimiento ( $\mathrm{R}$ ischard, 2003). Algo que, a partir de los datos obtenidos, parece no estar dándose, en medio del contexto de aproximación pasiva y meramente divulgativa que hacen las ONG, a través de las TIC; el cual debería avanzar hacia el aprovechamiento del amplio abanico de elementos que puedan favorecer la tecnología, a favor de la visibilidad y del impacto de la labor realizada por estas entidades. 


\section{Referencias}

ACEVEDO, M. Las TIC en las políticas de cooperación al desarrollo: hacia una nueva cooperación en la Sociedad Red. Cuadernos Internacionales de Tecnología para el Desarrollo Humano, n. 2, p. 1-10, 2004.

Integración de las tecnologías de la información y comunicación: asignatura pendiente de la cooperación. Madrid: CONGDE, 2006.

ALBAIGÈS, J. Usos y retos de las TIC en las organizaciones no lucrativas. Barcelona: Observatorio del Tercer Sector, 2007. AMBROSINI, M. Il terzo settore come fenomeno sociale: radicamento nella società e funzionamento organizzativo. In: Tra altruismo e professionalità. Milán: Angeli, 1999.

ARAYA, R. Internet, política y ciudadanía. Nueva Sociedad, n. 195, p. 56-71, 2005.

BARDÍN, L. Análisis de contenido. Madrid: Ediciones Akal, 1986.

BARONE, L. The 5 types of influencers on the Web, 2010. Disponible en: <http://smallbiztrends.com/2010/07/the5 -types-of-influencers-on-the-web.html>. Acceso en: 25 jun. 2012.

BERRIOS, O. Reflexiones, herramientas y experiencias 2.0 de cooperación. 2009. Disponible en: <http:// encuentro.1arroba1euro.org/reflexionesherramientasyexperiencias 20 decooperacionolgafernandezberrios.pdf $>$. Acceso en: 24 de jun. 2011.

BESSON, S.; MARTÍ, J. (Eds.). Deliberative democracy and its discontents: national and post-national challenges. Londres: Ashgate, 2006

BOHMAN, J.; REHG, W. (Eds.). Deliberative democracy: essays on reason and politics. Cambridge (Mass.): MIT Press, 1997.

CASTELLS, M. La era de la información: economía, sociedad y cultura. La sociedad en red. Madrid: Alianza, 2000, v. 1.

CARDOSO, G. Los medios de comunicación en la sociedad en red. Barcelona: UOC Ediciones, 2008.

COLEMAN, S.; GOTZE, J. Bowling together: online public engagement in policy deliberation. Londres: Hansard Society, 2001.

CEPAL. La sociedad de la información en América Latina y el Caribe: desarrollo de las tecnologías para el desarrollo. Santiago de Chile: Cepal, 2005.

CREMADES, J. Micropoder: la fuerza del ciudadano en la era digital. Madrid: Espasa-Calpe, 2007.

ELSTER, J. (Ed.). Deliberative democracy. Cambridge: Cambridge University Press, 1998.

GUI, B. Il ruolo delle organizzazioni mutualistiche e senza scopo di lucro. Bolonia: Il Mulino, 1990.
HAGEN, M. A tipology of electronic democracy. 1997. Disponible en: <http://www.uni-giessen.de/fb03/vinci/ labore/netz/hag_en.htm>. Acceso en: 19 mar. 2012.

HARTO, F. Tipologías y modelos de democracia electrónica. Revista de Internet, Derecho y Política, n. 2, p. 32-44, 2006.

HERRERA, M. La especificidad organizativa del tercer sector: tipos y dinámicas. Papers Revista de Sociología, n. 56, p. 163-196, 1998.

HOFF, J.; HORROCKS, I.; TOPS, P. (Eds.). Democratic governance and new technology. Londres: Routledge, 2000.

JOYANES, L. Cibersociedad: los retos sociales ante un nuevo mundo digital. Madrid: McGrawHill, 1997.

LABELLE, R. Information and Communication Technologies (ICTs) for Development in National Human Development Reports. Estados Unidos: PNUD, 2003.

MACEDO, S. (Ed.). Deliberative politics: essays on democracy and disagreement. Oxford: Oxford University Press, 1999. MARTÍ, J. Alguna precisión sobre las nuevas tecnologías y la democracia deliberativa y participativa. Revista de Internet, Derecho y Política, n. 6, p. 3-12, 2008.

MCCONNELL, P. (Ed.). Measuring the impact of information on development: overview of an international research program. Ottawa: IDRC, 1995.

NACIONES UNIDAS. Declaración del milenio. Estados Unidos: United Nations, 2000.

MORENO, F.; BRUQUETAS, M. Inmigración y estado de bienestar en España. Barcelona: Obra Social "La Caixa", 2010.

PEÑA-LOPEZ, I. Reticulando la cooperación-hacia la cooperación red: materiales para un debate. 2008. Disponible en: <http://ictlogy.net/articles/20080130_ismael_ pena_-_cooperacion_2.0.pdf>. Acceso en: 21 jun. 2011. PNUD. Informe sobre desarrollo humano 2001: poner el adelanto tecnológico al servicios del desarrollo humano. Estados Unidos: PNUD, 2001.

RISCHARD, J.-F. Integrating ICT in Development Programs. OECD Journal on Development, v. 4, n. 2, p. 5-22, 2003.

SÁNCHEZ, J. Perspectivas de la información en Internet: ciberdemocracia, redes sociales y web semántica. Revista Zer, v. 13, n. 25, p. 61-81, 2008.

SIERRA, F.; DEL VALLE, C. Nuevas tecnologías de la información y participación ciudadana. Redes.com, n. 4, p. 17-19, 2007.

SUBIRATS, J. Los dilemas de una relación inevitable. Innovación democrática y tecnologías de la información y de la comunicación. In: CAIRO, H. (Comp.). Democracia digital: límites y oportunidades. Madrid: Trotta, 2002. 


\title{
The use of social networks in NGO immigrants in Spain
}

\section{Abstract}

This article aims to analyze the use of social media by NGO working with immigrants in Spain. To do this, we analyzed 18 NGO, with Twitter and Facebook channels and web sites, as well as a total of 647 messages posted by them, during in 2010 and 2011. The data allow us to see how there is a low level of ownership of social networking by NGO analyzed, as well as informative and low participation in these scenarios that are employed today. These reasons reaffirm the Albaigès' words toward the lack of evidence for a timely use of the term " 2.0 cooperation" for the organizations surveyed.

Key words: social networks, NGO, immigration, participation, cooperation 2.0.

\section{O uso de redes sociais em ONGs imigrantes em Espanha}

\section{Resumo}

\begin{abstract}
Este artigo tem como objetivo investigar o uso das mídias sociais em ONGs que trabalham com imigrantes na Espanha. Para isso, foram pesquisadas 18 ONGs, tendo o Twitter e o Facebook como canais e portais web. Analisou-se um total de 647 mensagens postadas por elas, em 2010 e 2011. Os dados mostram um baixo nível de apropriação das redes sociais por parte dessas organizações, bem como uma baixa participação informativa nestes cenários em que estão empregadas, motivos para reafirmar as assertivas de Albaigès sobre a falta de elementos para uma utilização adequada do termo "cooperação 2.0" das ONGs em questão.
\end{abstract}

Palavras-chave: redes sociais, ONG, imigração, participação, cooperação 2.0.

Data de recebimento do artigo: 5/3/2013

Data de aprovação do artigo: 10/10/2013 